\title{
Comparison of Propofol and Ketofol on Transcranial Motor Evoked Potentials in Patients Undergoing Thoracolumbar Spine Surgery
}

\author{
Ankur Khandelwal ${ }^{1}$, Arvind Chaturvedi ${ }^{2}$, Navdeep Sokhal ${ }^{2}$, Akanksha Singh $^{3}$, Hanjabam Barun Sharma ${ }^{4}$ \\ ${ }^{1}$ Department of Anaesthesia and Critical Care, Sharda University School of Medical Sciences and Research, Greater Noida, India \\ ${ }^{2}$ Department of Neuroanaesthesiology and Critical Care, All India Institute of Medical Sciences, New Delhi, India \\ ${ }^{3}$ Department of Physiology, All India Institute of Medical Sciences, New Delhi, India \\ ${ }^{4}$ Department of Physiology, Institute of Medical Sciences, Banaras Hindu University, Varanasi, India
}

Study Design: This was a double-blind randomized study.

Purpose: The primary purpose was to compare the effects of propofol and ketofol on amplitudes and latencies of transcranial motor evoked potentials (TCMEPs) during thoracolumbar spine surgery. In addition, intraoperative hemodynamics and muscle power were compared.

Overview of Literature: Propofol is commonly used during intraoperative TcMEP monitoring. However, propofol inhibits TcMEP amplitudes and causes hypotension in a dose-dependent fashion.

Methods: Amplitude and latency of TcMEPs were recorded bilaterally from the abductor pollicis brevis (APB) and abductor hallucis (AH) muscles in 38 adult American Society of Anesthesiologists I and II patients undergoing thoracolumbar spine surgery. Baseline recordings of TCMEPs in both groups were recorded under propofol infusion. Group X patients then received propofol and fentanyl (1 mcg/ $\mathrm{kg} / \mathrm{hr})$, and group $Y$ patients received ketofol and fentanyl $(1 \mathrm{mcg} / \mathrm{kg} / \mathrm{hr})$. Bispectral index was maintained at 40-60 in both groups. Amplitude and latency were recorded at 30 minutes intervals for 2 hours.

Results: Propofol caused no significant changes in amplitude and latency in any muscle. In contrast, amplitude increased significantly at all time points in the bilateral APB muscles and 60, 90, and 120 minutes in the left AH muscle without changes in latency in response to ketofol. When the two groups were compared, ketofol induced significantly higher amplitudes at 60, 90, and 120 minutes in the (left) APB, at all time points in the (right) APB, and at 120 minutes in both AH muscles, compared with propofol. Blood pressures were lower and fluid and vasopressor requirements were higher in group X. Muscle power was similar between the two groups. Conclusions: Ketofol facilitates TcMEP amplitudes without affecting latency. Use of ketofol resulted in a better and more stable hemodynamic profile than did use of propofol.

Keywords: Amplitude; Ketofol; Latency; Muscle power; Propofol; Transcranial motor evoked potentials

Received Feb 27, 2021; Revised Feb 27, 2021; Accepted Mar 8, 2021

Corresponding author: Arvind Chaturvedi

Department of Neuroanaesthesiology and Critical Care, All India Institute of Medical Sciences, New Delhi, 110029 India

Tel: +91-011-26593784, Fax: +91-011-26588663, E-mail: nscanaesthesia@aiims.edu 


\section{Introduction}

Intraoperative neurophysiological monitoring (IONM) is the standard of care during spine surgeries. IONM provides real-time feedback of impending or potential new neurological deficits and the opportunity for corrective action before an insult causes permanent neurological damage $[1,2]$. However, the diagnostic performance of IONM depends on selecting the appropriate monitoring techniques, based on surgical, anesthetic, and patientrelated factors [2]. Transcranial motor evoked potentials (TcMEPs) effectively monitor the integrity of the motor pathway during spine surgery with a sensitivity and specificity as high as $100 \%$ and $95 \%-100 \%$, respectively $[3,4]$. However, successful TcMEP monitoring is critically dependent on the anesthetic technique; some anesthetic agents suppress TcMEP signals at commonly used concentrations, leading to inconsistent TcMEP recordings. Total intravenous anesthetics (TIVAs) are superior to inhalational agents for TcMEP monitoring [5]. TIVA, using propofol and a synthetic narcotic, is the most commonly used anesthetic regimen for TcMEP monitoring [6]. However, propofol inhibits TcMEP amplitude in a dose-dependent fashion [6,7]. Moreover, high dose propofol is associated with hypotension; thus, propofol is unsuitable for patients with hemodynamic and cardiovascular instability.

Ketamine [8], dexmedetomidine [9], and lidocaine [10] have been used as adjuvants to reduce propofol requirements with variable effects on TcMEPs. The ketaminepropofol admixture (ketofol) has been used successfully in various procedures and produces rapid onset of sedation, appropriate hemodynamic conditions, and rapid recovery $[11,12]$. However, no study has evaluated the effects of ketofol infusion (ketamine-propofol, 1:4 admixture) on TcMEPs during general anesthesia maintenance.

The primary objective of this study was to compare the effects of propofol versus ketofol on amplitudes and latencies of TcMEPs, when these agents are used with fentanyl for the maintenance of general anesthesia during thoracolumbar spine surgery. The secondary objectives included comparing intraoperative hemodynamic parameters between the two anesthetic regimens and comparing muscle power at hospital discharge. We hypothesized that the addition of low-dose ketamine to propofol TIVA would exert a favorable effect on TcMEPs.

\section{Materials and Methods}

\section{Study design}

In this study, 38 patients undergoing elective lower thoracic (below T5) and lumbar spine surgery with TcMEP monitoring were enrolled. Approval was obtained from the Institutional Ethics Committee of All India Institute of Medical Sciences, New Delhi (IECPG-594/20.12.2017, RT-71/31.01.2018), and written informed consent was obtained from all patients. The study is registered in the Clinical Trial Registry of India (REF/2017/12/016146).

Inclusion criteria included age between 18 and 65 years, American Society of Anesthesiologists (ASA) physical statuses I and II, upper extremity muscle power 5 (Medical Research Council Muscle Power Grading), and lower extremity muscle power $\geq 3$. The exclusion criteria included the patient's refusal to consent, upper extremity muscle power $<5$ or lower extremity muscle power $<3$, pre-existing myopathies, morbid obesity, and a history of coronary artery disease, chronic renal and hepatic insufficiency, allergies to any study medications, seizures, or psychiatric disease.

\section{Sample size determination}

Sample size was calculated to detect effect size for mean amplitude or latency between the two groups up to 0.9 per standard deviation (SD) or more, with $95 \%$ confidence levels and $80 \%$ power. The calculated sample size was 19 evaluable patients per group. Thus, 38 patients were enrolled in the study.

\section{Randomization, group allocation, and intervention}

Sixty patients were assessed for eligibility, and 38 patients met the inclusion criteria (Fig. 1). The patients were allocated into two groups in a 1:1 ratio using computergenerated block randomization. Opaque sealed envelopes were used for group allocation, and the study drugs were prepared by an independent neuroanesthesiologist who was not involved in administering the drugs or the postoperative patient follow-up. All study medications were prepared in identical $50 \mathrm{~mL}$ disposable syringes. For group $\mathrm{X}$, a $50 \mathrm{~mL}$ syringe was filled with $1 \%$ propofol (10 $\mathrm{mg} / \mathrm{mL}$ ). For group Y, ketofol solution (total $50 \mathrm{~mL}$ ) was prepared by adding $2 \mathrm{~mL}$ of ketamine (100 mg; $50 \mathrm{mg} /$ $\mathrm{mL}), 40 \mathrm{~mL}$ of $1 \%$ propofol $(400 \mathrm{mg} ; 10 \mathrm{mg} / \mathrm{mL})$, and 8 


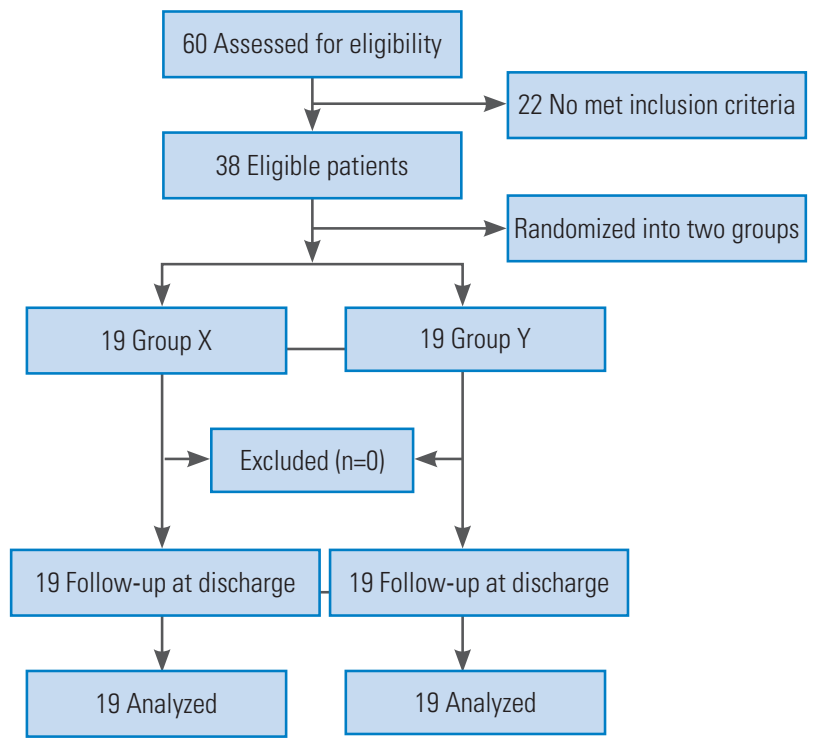

Fig. 1. CONSORT flow diagram.

$\mathrm{mL}$ of saline. Thus, the effective concentration of ketamine $(2 \mathrm{mg} / \mathrm{mL})$ and propofol $(8 \mathrm{mg} / \mathrm{mL})$ was $1: 4(\mathrm{mg})$ in the prepared solution. Fentanyl was prepared in a separate 50 $\mathrm{mL}$ syringe containing $10 \mathrm{mcg} / \mathrm{mL}$ of fentanyl.

All participants were pre-medicated with intramuscular glycopyrrolate $(0.2 \mathrm{mg}) 30$ minutes before surgery. In the operating room, standard ASA monitors, including noninvasive blood pressure, electrocardiogram, and pulse oximeter $\left(\mathrm{SpO}_{2}\right)$, were attached to the patients. Bispectral index (BIS) sensors were applied to the patients' forehead to monitor the depth of anesthesia. After pre-oxygenation with $100 \%$ oxygen, general anesthesia was induced with fentanyl $(2 \mathrm{mcg} / \mathrm{kg})$ and propofol $(2-2.5 \mathrm{mg} / \mathrm{kg})$ titrated to loss of verbal command. Cisatracurium $(0.15 \mathrm{mg} / \mathrm{kg})$ was administered to facilitate tracheal intubation. An arterial line was inserted, and a temperature probe was placed in the nasopharynx for intraoperative temperature monitoring. A soft bite block was placed to prevent tongue bite and oral cavity injuries secondary to TcMEP stimulation. Mechanical ventilation was carried out at a fresh gas flow of $2 \mathrm{~L} / \mathrm{min}$ with oxygen and air $(0.5$ fraction of inspired oxygen). Tidal volume was fixed at $8 \mathrm{~mL} / \mathrm{kg}$, and respiratory rate was adjusted to maintain the partial pressure of carbon dioxide between 35 and $40 \mathrm{~mm} \mathrm{Hg}$. Anesthesia maintenance was commenced with intravenous (IV) infusions of fentanyl $(1 \mathrm{mcg} / \mathrm{kg} / \mathrm{hr})$ and propofol titrated to maintain BIS at 40-60. A threshold voltage of TcMEP was recorded once T4/T1 (train-of-four [TOF] stimulation test, neuromuscular monitoring) was more than $75 \%$. Af- ter the threshold voltage was obtained, stimulation voltage was given at $10 \%$ above the threshold for eliciting baseline TcMEP parameters (amplitude in microvolts and latency in milliseconds) in all patients. Once the baseline amplitude and latency of the monitored muscles were recorded, anesthesia was maintained in each group. In group X, anesthesia was maintained with IV infusions of fentanyl $(1 \mathrm{mcg} / \mathrm{kg} / \mathrm{hr}$ ) and propofol titrated to maintain BIS at 40-60. In group $\mathrm{Y}$, anesthesia was maintained with IV infusions of fentanyl $(1 \mathrm{mcg} / \mathrm{kg} / \mathrm{hr})$ and ketofol titrated to maintain BIS at 40-60. In both groups, mean arterial pressure (MAP) was maintained within $20 \%$ of baseline values. If hypotension (decrease in MAP $>20 \%$ of baseline) developed, a fluid bolus $(200 \mathrm{~mL})$ was administered. If hypotension persisted after the fluid bolus, phenylephrine boluses in incremental doses of $20 \mathrm{mcg}$ were administered to maintain MAP. If hypertension (increase in MAP $>20 \%$ of baseline) developed, labetalol (5 mg) was considered. Intermittent fentanyl boluses $(0.5 \mathrm{mcg} / \mathrm{kg})$ were administered at the discretion of the attending neuroanesthesiologist. Intraoperative body temperature was maintained between $36^{\circ} \mathrm{C}$ and $37^{\circ} \mathrm{C}$.

\section{Acquisition of transcranial motor evoked potentials}

TcMEP responses were elicited after transcranial electrical stimulation through corkscrew electrodes placed at C3 and C4 positions according to the 10-20 electrode placement system. Stimulation consisted of a train of eight square wave pulses of $75 \mu$ duration each, with a frequency of 250-500 pulses/sec (software version NIM ECLIPSE 4.0, NIM ECLIPSE NS System; Medtronic, Minneapolis, MN, USA). TcMEPs were recorded bilaterally from the abductor pollicis brevis (APB) muscle (control muscle) of the upper limbs and abductor hallucis (AH) muscle (affected muscle) of the lower limbs through paired needle electrodes.

The effects of muscle relaxants were assessed using the TOF modality. The threshold voltage was recorded once $\mathrm{T} 4 / \mathrm{T} 1$ was more than $75 \%$. After determining the threshold voltage, the stimulation voltage was given at $10 \%$ above threshold for eliciting baseline (T0) MEP parameters (amplitude and latency). The same stimulation voltage was used in each respective patient throughout the surgery for recording amplitude and latency at 30 minutes intervals for 2 hours; the 30-, 60-, 90-, and 120-minute time points are labeled as $\mathrm{T} 1, \mathrm{~T} 2, \mathrm{~T} 3$, and $\mathrm{T} 4$, respectively. 


\section{Blinding}

The patients, investigator, and neurophysiologist were blinded to the anesthetic regimen.

\section{Statistical analysis}

Data were analyzed using IBM SPSS ver. 25.0 (IBM Corp., Armonk, NY, USA). Normality was determined using the Shapiro-Wilk test. Descriptive statistics were determined. Normally distributed or parametric data are presented as mean $\pm \mathrm{SD}$, whereas non-normally distributed or nonparametric data are presented as medians (quartile difference $[Q D])$. Parameters were compared within the same group over time using repeated-measures analysis of variance and the Friedman test for parametric and nonparametric data, respectively. Bonferroni post hoc test was applied when $p$-values were significant. Parameters were compared between the two groups using unpaired $t$ tests or Mann-Whitney $U$-tests for parametric and nonparametric data, respectively. The statistical significance threshold was $p<0.05$.

\section{Results}

\section{Demographics and spinal pathology}

There were no significant differences between the two groups regarding sex, age, height, or weight (Table 1). Spinal disorders included spinal cord tumor $(n=22)$, spinal canal stenosis $(n=7)$, disc herniation $(n=5)$, lumbar listhesis $(n=3)$, and ossification of the posterior longitudinal ligament $(n=1)$.

\section{Transcranial motor evoked potentials parameters}

No significant changes in amplitude were detected over

Table 1. Table showing comparison of demographic variables between the two groups

\begin{tabular}{lccc} 
Variable & Group X & Group Y & $p$-value \\
Age $(\mathrm{yr})$ & $41.11 \pm 14.72$ & $42.05 \pm 14.65$ & $0.843^{\mathrm{a})}$ \\
Weight $(\mathrm{kg})$ & $65.21 \pm 10.54$ & $63.79 \pm 8.55$ & $0.651^{\text {a) }}$ \\
\hline Height $(\mathrm{cm})$ & $170(3)$ & $170(5)$ & $0.781^{\text {b) }}$ \\
\hline
\end{tabular}

Values are presented as mean \pm standard deviation or median (quartile difference).

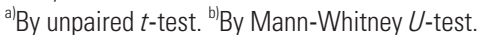

time in the bilateral APB (Fig. 2) and AH muscles in group $\mathrm{X}$. In group $\mathrm{Y}$, amplitude increased at all time points compared with baseline in the bilateral APB muscles (Fig. 3) and at T2, T3, and T4 versus T0 in the AH (left [L]) muscle. In the AH (right $[\mathrm{R}]$ ) muscle, no significant increases in amplitude in T1-T4 compared with baseline (T0) were detected (Fig. 4). No significant changes in amplitude were detected between any successive time points (T1-T4) in either group (Table 2).

Amplitudes in group $\mathrm{Y}$ were significantly higher in the APB (L) at T2, T3, and T4 and in the APB (R) at all time points compared with those in group $\mathrm{X}$. In the bilateral $\mathrm{AH}$ muscles, the amplitudes were higher at all time points in group $\mathrm{Y}$ compared with those in group $\mathrm{X}$, though the differences were only significant at T4 (Table 3 ).

There were no significant differences in latency at any time points (T1-T4) compared with baseline (T0) in

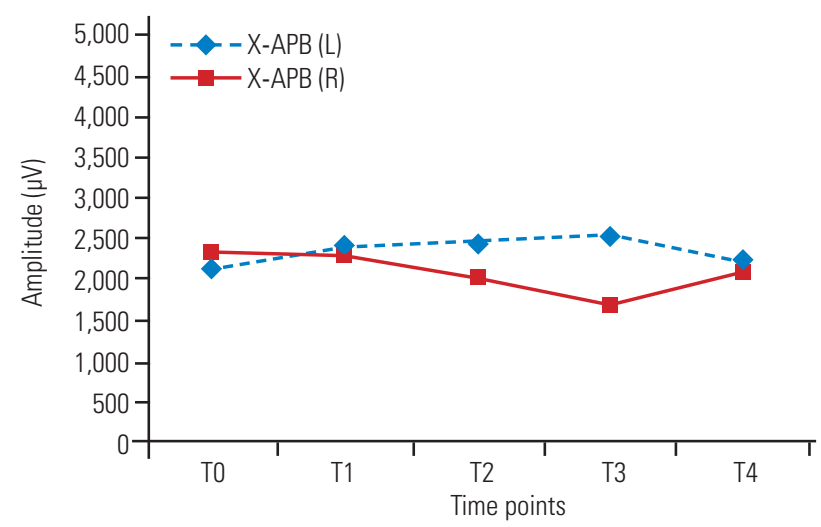

Fig. 2. Line diagram showing trends of amplitude $(\mu \mathrm{V})$ of $A P B(L)$ and $A P B(R)$ in group $X$ across various time points. Values are presented as median. APB, abductor pollicis brevis; L, left; R, right.

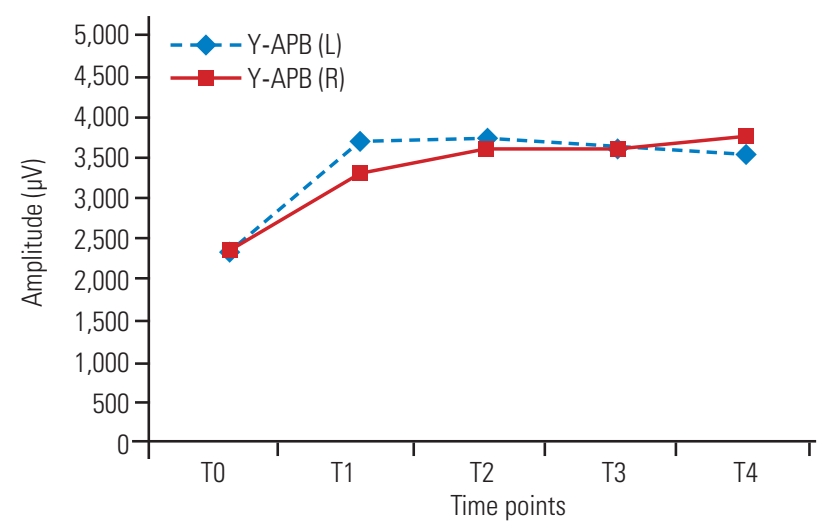

Fig. 3. Line diagram showing trends of amplitude $(\mu \mathrm{V})$ in $A P B(L)$ and $A P B(R)$ in group $Y$ across various time points. Values are presented as median. APB, abductor pollicis brevis; $L$, left; $R$, right. 
either group (Table 4). When the two groups were compared, the latency was similar at all time points (Table 5).

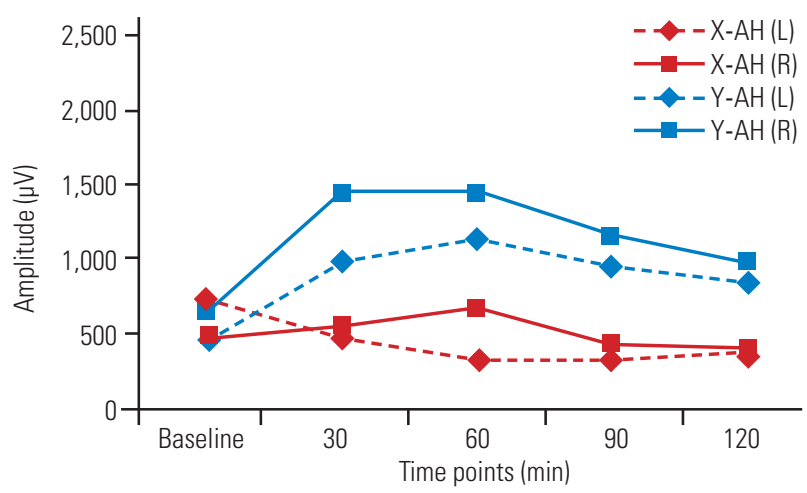

Fig. 4. Line diagram showing trends of amplitude $(\mu \mathrm{V})$ in $A H(L)$ and $A H(R)$ in group $\mathrm{X}$ and group $\mathrm{Y}$ across various time points. Values are presented as median. AH, abductor hallucis; L, left; $R$, right.

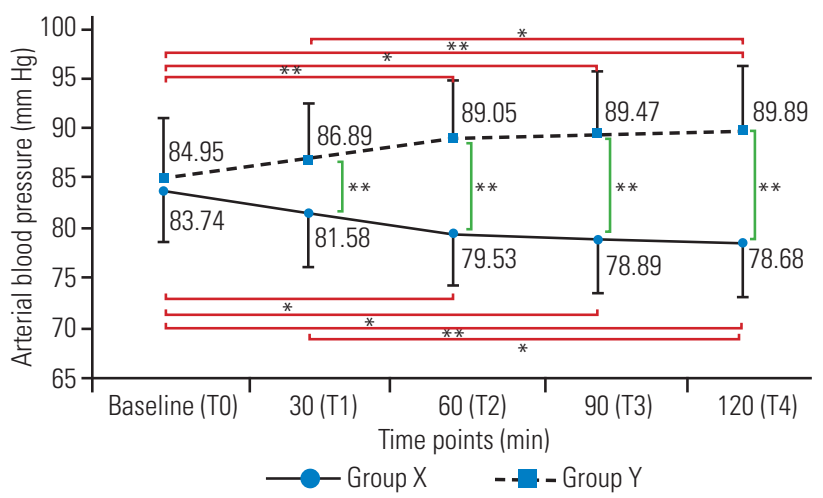

Fig. 5. Line diagram showing the trends of mean arterial blood pressure in the two groups across different time points. Values are presented as mean \pm standard deviation. ${ }^{*} p<0.05 .{ }^{* *} p<0.01$.
In group $\mathrm{X}, \mathrm{MAP}$ decreased significantly at $\mathrm{T} 2$ (mean \pm SD, $79.53 \pm 5.26 \mathrm{~mm} \mathrm{Hg})$, T3 $(78.89 \pm 5.47 \mathrm{~mm}$ $\mathrm{Hg})$, and $\mathrm{T} 4(78.68 \pm 5.48 \mathrm{~mm} \mathrm{Hg})$ compared with $\mathrm{T} 0$ $(83.74 \pm 5.22 \mathrm{~mm} \mathrm{Hg})$. In group Y, MAP increased significantly at T2 $(89.05 \pm 6.38 \mathrm{~mm} \mathrm{Hg}), \mathrm{T} 3(89.47 \pm 6.17$ $\mathrm{mm} \mathrm{Hg})$, and $\mathrm{T} 4(89.89 \pm 6.33 \mathrm{~mm} \mathrm{Hg})$ compared with T0 $(84.95 \pm 6.09 \mathrm{~mm} \mathrm{Hg})$. MAP was similar between the two groups at T0. However, at all other time points, BP was significantly higher in group Y compared with that in group X (Fig. 5). No significant differences in HR were detected within the two groups or between the two groups (Fig. 6).

\section{Intraoperative parameters}

BIS values did not change significantly in group $\mathrm{X}$ at any time points compared with baseline (T0). In group Y,

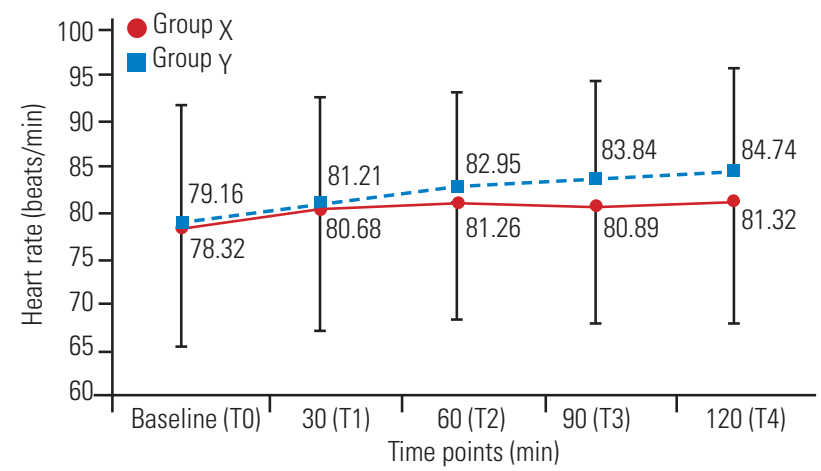

Fig. 6. Line diagram showing the trends of mean heart rate in the two groups across different time points. Values are presented as mean \pm standard deviation. ${ }^{*} p<0.05$. ${ }^{* *} p<0.01$.

Table 2. Table showing trend of amplitude $(\mu \mathrm{V})$ within the group at different time points ${ }^{\text {a) }}$

\begin{tabular}{|c|c|c|c|c|c|c|c|c|}
\hline \multirow{2}{*}{ Time point } & \multicolumn{4}{|c|}{ Group X } & \multicolumn{4}{|c|}{ Group Y } \\
\hline & APB (L) & APB (R) & $\mathrm{AH}(\mathrm{L})$ & $\mathrm{AH}(\mathrm{R})$ & APB (L) & APB (R) & $\mathrm{AH}(\mathrm{L})$ & $\mathrm{AH}(\mathrm{R})$ \\
\hline Amplitude (TO) & $2,167(1,003)$ & $2,347(893.5)$ & $729(517.5)$ & $482(523)$ & $2,272.2 \pm 932.9$ & $2,295.68 \pm 1,016.2$ & $461(219.5)$ & 650 (351.5) \\
\hline Amplitude (T1) & $2,410(1,345)$ & $2,304(1,141.5)$ & $474(477.5)$ & $549(530)$ & $3,621.05 \pm 1,277.6^{* *}$ & $3,221.21 \pm 704.181^{*}$ & $990(610.5)$ & $1,450(682)$ \\
\hline Amplitude (T2) & 2,438 (836.5) & 2,010 (323.5) & 341 (443.5) & $661(670)$ & $3,675.7 \pm 1,309.5^{* *}$ & $3,535.16 \pm 1,220.06^{* *}$ & $1,145.00^{*}(615.5)$ & $1,450(845)$ \\
\hline Amplitude (T3) & $2,523(562.5)$ & $1,695(787)$ & $333(448.5)$ & $427(571.5)$ & $3,531.5 \pm 1,300.2^{* *}$ & $3,538.89 \pm 1,061.93^{* *}$ & $950.00^{*}(519.5)$ & $1,157(504)$ \\
\hline Amplitude (T4) & $2,238(756.5)$ & $2,078(1,269)$ & $378(289.5)$ & $413(664)$ & $3,450.9 \pm 1,096.8^{* *}$ & $3,684.47 \pm 986.98^{* *}$ & $848.00^{* *}(511)$ & 978 (403.5) \\
\hline$p$-value & 0.927 & 0.824 & 0.057 & 0.098 & $<0.001^{* * *}$ & $<0.001^{* * *}$ & $0.005^{*}$ & 0.143 \\
\hline Test & FT & FT & FT & FT & RA & RA & $\mathrm{FT}$ & FT \\
\hline
\end{tabular}

Values are presented as median (QD) or mean \pm SD.

APB, abductor pollicis brevis; AH, abductor hallucis; L, left; R, right; FT, Friedman test; RA, repeated measures analysis of variance; $Q D$, quartile difference; SD, standard deviation.

${ }^{*} p<0.05,{ }^{* *} p<0.01$, and ${ }^{* * *} p<0.001$ when compared to baseline. ${ }^{\text {al }} \mathrm{FT}$ with Bonferroni post hoc test and RA with Bonferroni post hoc tests were applied for median (QD) and mean $\pm S D$, respectively. 


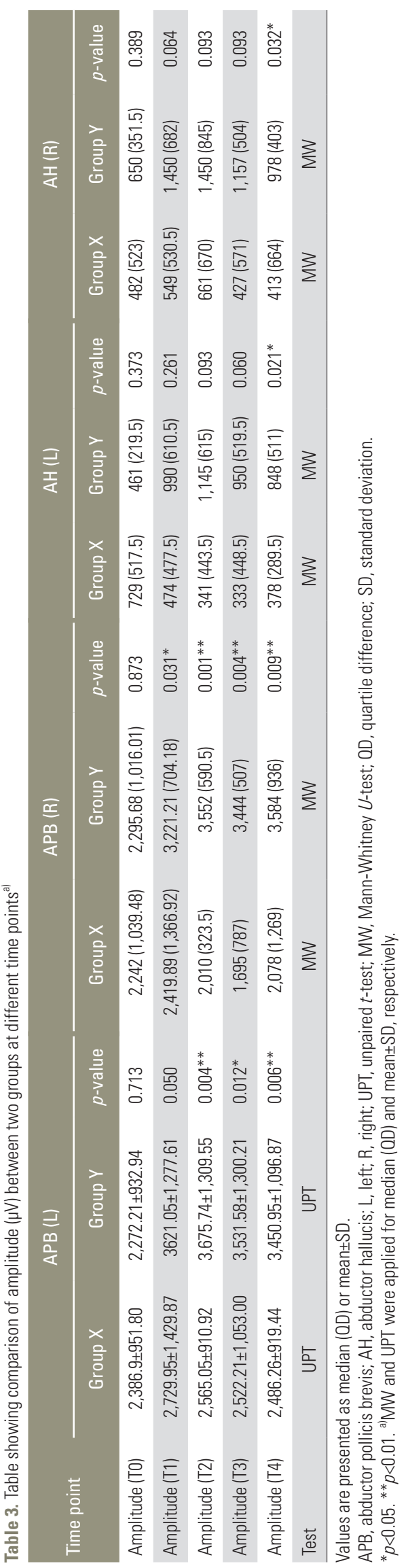

BIS increased significantly at all time points compared with baseline; however, there were no significant changes among the time points (T1 to T4). BIS was similar at baseline between the two groups; however, BIS was significantly higher at all other time points in group $\mathrm{Y}$ compared with that in group X (Table 6). BIS was always within the range of 40-60 in both groups at all time points. The mean consumption of IV fluids $(3,005.26 \pm 605.96 \mathrm{~mL}$ in group X versus $2,594.74 \pm 402.04 \mathrm{~mL}$ in group $\mathrm{Y}$ ) and phenylephrine use $(178.95 \pm 66.82 \mathrm{mcg}$ in group $\mathrm{X}$ versus $90.53 \pm 61.23 \mathrm{mcg}$ in group Y) were significantly higher in group X compared with that in group Y. The mean blood volume loss, median blood transfusion volume, surgery duration, and anesthesia duration were similar between the two groups. Blood transfusions were required in five patients in group $\mathrm{X}(300 \mathrm{~mL}$ each) and four patients in group Y (300 mL each). The median (QD) time to tracheal extubation was significantly shorter in group $\mathrm{X}$ compared with that in group Y (20 [2] minutes versus 28 [3.5] minutes) (Table 7).

\section{Complications}

One patient in group $\mathrm{Y}$ developed intraoperative hypertension, which was treated with labetalol $(5 \mathrm{mg})$. One patient in group Y suffered a tongue laceration secondary to TcMEP stimulation. No other patients developed major intraoperative and postoperative complications. None of the patients in group Y developed ketamine-induced psychedelic effects during the postoperative period.

There were no significant differences in muscle power preoperatively versus postoperatively in either the upper or lower limb muscles in the two groups (Table 8). The median (QD) duration of hospital stays (from the day of surgery till the day of discharge) was similar between the two groups (4 [1] and 4 [1] in groups $\mathrm{X}$ and $\mathrm{Y}$, respectively; $p=0.751)$.

\section{Discussion}

TcMEPs were recorded successfully in all patients. The effects of ketofol on TcMEPs have not been investigated to our knowledge; thus, we compared our results with those studies that utilized ketamine as an adjuvant with propofol TIVA during TcMEP monitoring.

No significant changes in amplitude and latency in the bilateral APB and AH muscles over time were observed 
with propofol. However, with ketofol, amplitude increased significantly at all time points in the bilateral APB muscles and at 60,90 , and 120 minutes in the $\mathrm{AH}(\mathrm{L})$ muscle compared with baseline, without changes in latency. In the $\mathrm{AH}$

Table 4. Table showing trend of latency (ms) within the group at different time points ${ }^{\text {a) }}$

\begin{tabular}{|c|c|c|c|c|c|c|c|c|}
\hline \multirow{2}{*}{ Time point } & \multicolumn{4}{|c|}{ Group X } & \multicolumn{4}{|c|}{ Group Y } \\
\hline & APB (L) & APB (R) & $\mathrm{AH}(\mathrm{L})$ & $\mathrm{AH}(\mathrm{R})$ & APB (L) & APB (R) & $\mathrm{AH}(\mathrm{L})$ & $\mathrm{AH}(\mathrm{R})$ \\
\hline Latency (TO) & $27.56 \pm 4.05$ & $27.09 \pm 2.22$ & $44.79 \pm 7.42$ & $43.88 \pm 6.71$ & $27.80(2.3)$ & $27.50(2.65)$ & $45.58 \pm 9.33$ & $45.17 \pm 9.54$ \\
\hline Latency (T1) & $27.55 \pm 3.74$ & $27.11 \pm 2.63$ & $44.18 \pm 6.54$ & $42.17 \pm 6.86$ & $27.60(3.05)$ & $27.40(2.1)$ & $43.61 \pm 6.88$ & $44.42 \pm 9.05$ \\
\hline Latency (T2) & $27.58 \pm 3.43$ & $27.15 \pm 2.48$ & $44.59 \pm 7.45$ & $43.15 \pm 7.01$ & $26.60(3.25)$ & $27.30(2.00)$ & $43.29 \pm 7.00$ & $43.89 \pm 8.9$ \\
\hline Latency (T3) & $27.81 \pm 3.72$ & $27.20 \pm 2.60$ & $43.99 \pm 6.75$ & $43.45 \pm 7.51$ & $27.60(3.45)$ & $26.70(1.95)$ & $42.26 \pm 6.64$ & $44.15 \pm 9.8$ \\
\hline Latency (T4) & $28.02 \pm 3.72$ & $27.12 \pm 2.86$ & $44.55 \pm 7.22$ & $42.67 \pm 7.21$ & $27.20(2.7)$ & $27.40(1.85)$ & $42.49 \pm 5.22$ & $43.67 \pm 8.78$ \\
\hline$p$-value & 0.686 & 0.999 & 0.753 & 0.15 & 0.146 & 0.902 & 0.059 & 0.161 \\
\hline Test & RA & RA & RA & RA & $\mathrm{FT}$ & $\mathrm{FT}$ & $\mathrm{RA}$ & RA \\
\hline
\end{tabular}

Values are presented as mean \pm SD or median (OD).

APB, abductor pollicis brevis; AH, abductor hallucis; L, left; R, right; RA, repeated measures analysis of variance; FT, Friedman test; SD, standard deviation; QD, quartile difference.

${ }^{\text {a) }}$ T and RA tests were applied for median (OD) and mean $\pm S D$, respectively.

Table 5. Table showing comparison of latency (ms) between the two groups ${ }^{\text {a) }}$

\begin{tabular}{|c|c|c|c|c|c|c|c|c|c|c|c|c|c|c|}
\hline \multirow{2}{*}{$\begin{array}{l}\text { Time } \\
\text { period }\end{array}$} & \multicolumn{3}{|c|}{ APB (L) } & \multirow{2}{*}{ Test } & \multicolumn{3}{|c|}{ APB (R) } & \multicolumn{3}{|c|}{$\mathrm{AH}(\mathrm{L})$} & \multicolumn{3}{|c|}{$\mathrm{AH}(\mathrm{R})$} & \multirow{2}{*}{ Test } \\
\hline & Group X & Group Y & $p$-value & & Group X & Group Y & $p$-value & Group X & Group Y & $p$-value & Group X & Group Y & $p$-value & \\
\hline Latency (TO) & $27.80(3.45)$ & $27.80(2.3)$ & 0.726 & MW & $27.09 \pm 2.28$ & $27.85 \pm 2.95$ & 0.390 & $44.79 \pm 7.42$ & $45.58 \pm 9.33$ & 0.775 & $43.88 \pm 6.71$ & $45.71 \pm 9.54$ & 0.631 & UPT \\
\hline Latency (T1) & $27.20(2.95)$ & 27.60 (3.05) & 0.726 & MW & $27.11 \pm 2.63$ & $27.28 \pm 3.29$ & 0.859 & $44.18 \pm 6.54$ & $43.61 \pm 6.88$ & 0.792 & $42.17 \pm 6.86$ & $44.42 \pm 9.05$ & 0.393 & UPT \\
\hline Latency (T2) & $27.40(2.5)$ & 26.60 (3.25) & 0.930 & MW & $26.80 \pm 1.3$ & $27.30 \pm 2$ & 0.804 & $44.59 \pm 7.45$ & $43.29 \pm 7.00$ & 0.581 & $43.15 \pm 7.01$ & $43.89 \pm 8.90$ & 0.777 & UPT \\
\hline Latency (T3) & $28.00(3.9)$ & $27.60(3.45)$ & 0.942 & MW & $27.10 \pm 1.1$ & $26.70 \pm 1.95$ & 0.977 & $43.99 \pm 6.75$ & $42.26 \pm 6.64$ & 0.431 & $43.45 \pm 7.51$ & $44.15 \pm 9.80$ & 0.806 & UPT \\
\hline Latency (T4) & $28.60(3.5)$ & $27.20(2.7)$ & 0.405 & MW & $27.10(1.35)$ & $27.40(3.7)$ & 0.704 & 44.55 (7.22) & 42.49 (5.22) & 0.321 & 40.00 (3.9) & $42.10(3.6)$ & 0.511 & MW \\
\hline
\end{tabular}

Values are presented as median (OD) or mean \pm SD, unless otherwise stated.

APB, abductor pollicis brevis; AH, abductor hallucis; L, left; R, right; MW, Mann-Whitney U-test; UPT, unpaired $t$-test; $Q D$, quartile difference; SD, standard deviation.

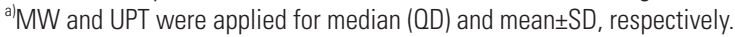

Table 6. Table showing BIS comparison within and across the groups across different time points ${ }^{\text {al }}$

\begin{tabular}{|c|c|c|c|c|c|c|}
\hline \multirow{2}{*}{ Time period } & \multicolumn{2}{|c|}{ Group X } & \multicolumn{2}{|c|}{ Group Y } & \multirow{2}{*}{$\begin{array}{c}p \text {-value } \\
\text { (across the group) }\end{array}$} & \multirow{2}{*}{ Test } \\
\hline & Mean \pm SD & Median (QD) & $\operatorname{Mean} \pm \mathrm{SD}$ & Median (QD) & & \\
\hline $\mathrm{BIS}(\mathrm{TO})$ & $46.42 \pm 4.06$ & $46(2.25)$ & $46 \pm 4.22$ & $46(4)$ & 0.756 & UPT \\
\hline $\mathrm{BIS}(\mathrm{T} 1)$ & $45.84 \pm 3.934$ & $45(3)$ & $55.11 \pm 2.78$ & $56(2.5)$ & $<0.001^{* *}$ & MW \\
\hline $\mathrm{BIS}(\mathrm{T} 2)$ & $47.00 \pm 3.712$ & $48(3)$ & $55.26 \pm 2.75$ & $56(2)$ & $<0.001^{* *}$ & MW \\
\hline $\mathrm{BIS}(\mathrm{T} 3)$ & $46.79 \pm 3.824$ & $48(3)$ & $55.58 \pm 2.46$ & $56(1.5)$ & $<0.001^{* *}$ & MW \\
\hline $\mathrm{BIS}(\mathrm{T} 4)$ & $47.68 \pm 3.497$ & $48(3)$ & $56.47 \pm 2.23$ & $56(1)$ & $<0.001^{* *}$ & MW \\
\hline$p$-value (within the group) & \multicolumn{2}{|c|}{0.113} & \multicolumn{2}{|c|}{$<0.001^{* *}$} & & \\
\hline Test & \multicolumn{2}{|c|}{ RA } & \multicolumn{2}{|c|}{$\mathrm{FT}$} & & \\
\hline
\end{tabular}

Values are presented as mean \pm SD or median (OD).

BIS, Bispectral index; SD, standard deviation; QD, quartile difference; UPT, unpaired $t$-test; MW, Mann-Whitney U-test; RA, repeated measures analysis of variance;

FT, Friedman test.

${ }^{* *} p<0.01$. ${ }^{\text {a) }}$ T with Bonferroni post hoc test and MW test were applied for median (QD); RA and UPT tests were applied for mean \pm SD. 
Table 7. Table showing comparison of various intraoperative parameters between the two groups ${ }^{\mathrm{a}}$

\begin{tabular}{|c|c|c|c|c|}
\hline Variable & Group X & Group Y & $p$-value & Test \\
\hline Intravenous fluid (mL) & $3,005.26 \pm 605.96$ & $2,594.74 \pm 402.04$ & $0.019^{*}$ & UPT \\
\hline Blood loss (mL) & $455.26 \pm 180.20$ & $426.32 \pm 149.41$ & 0.593 & UPT \\
\hline Phenylephrine consumption (mcg) & $178.95 \pm 66.82$ & $90.53 \pm 61.23$ & $<0.001^{* *}$ & UPT \\
\hline Propofol consumption (mg) & $1,118.42 \pm 165.17$ & $607.89 \pm 88.61$ & $<0.001^{* *}$ & UPT \\
\hline Duration of surgery (min) & $227.37 \pm 40.53$ & $213.68 \pm 33.53$ & 0.264 & UPT \\
\hline Duration of anesthesia (min) & $272.11 \pm 40.77$ & $274.74 \pm 30.44$ & 0.823 & UPT \\
\hline Blood transfusion (mL) & $0.00(150)$ & $0.00(0)$ & 0.467 & MW \\
\hline Extubation time (min) & $20(2)$ & $28(3.5)$ & $<0.001^{* *}$ & MW \\
\hline
\end{tabular}

Values are presented as mean $\pm S D$ or median (OD).

UPT, unpaired $t$-test; MW, Mann-Whitney $U$-test; SD, standard deviation; OD, quartile difference.

${ }^{*} p<0.05 .{ }^{* *} p<0.01$. ${ }^{\text {al }} \mathrm{MW}$ and UPT were applied for median (OD) and mean $\pm \mathrm{SD}$, respectively.

Table 8. Table showing comparison of muscle power preoperatively versus postoperatively in both groups ${ }^{\text {a) }}$

\begin{tabular}{|c|c|c|c|c|c|c|c|c|}
\hline \multirow{3}{*}{ Group } & \multicolumn{8}{|c|}{ Muscle power } \\
\hline & \multicolumn{2}{|c|}{ APB (L) $(n=19)$} & \multicolumn{2}{|c|}{ APB (R) $(n=19)$} & \multicolumn{2}{|c|}{$A H(L)(n=19)$} & \multicolumn{2}{|c|}{$\mathrm{AH}(\mathrm{R})(\mathrm{n}=19)$} \\
\hline & Preop & Postop & Preop & Postop & Preop & Postop & Preop & Postop \\
\hline Group X & $5.00(0)$ & $5.00(0)$ & $5.00(0)$ & $5.00(0)$ & $4.00(1)$ & $4.00(1)$ & $4.00(1)$ & $4.00(1)$ \\
\hline Group Y & $5.00(0)$ & $5.00(0)$ & $5.00(0)$ & $5.00(0)$ & $4.00(1)$ & $4.00(1)$ & $4.00(1)$ & $4.00(1)$ \\
\hline$p$-value & 1.00 & 1.00 & 1.00 & 1.00 & 0.691 & 0.244 & 0.481 & 0.370 \\
\hline
\end{tabular}

Values are presented as median (quartile difference).

APB, abductor pollicis brevis; $A H$, abductor hallucis; $L$, left; $R$, right; Preop, preoperative; Postop, postoperative.

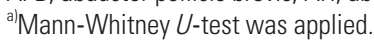

(R) muscle, there was no significant increase in amplitude from $\mathrm{T} 1$ to $\mathrm{T} 4$ compared with baseline (T0). Also, there were no significant changes in amplitude between the times after baseline (T1-T4) in group Y, indicating that ketofol maintained a stable depth of anesthesia, which is the prime requirement for accurate intraoperative neurophysiological interpretation of TcMEPs. Ketamine increases muscle tone and could well be the reason for the ketofol induced increased amplitudes, as we did not administer muscle relaxant during maintenance of anesthesia [13].

Lam et al. [14] observed that ketamine infusion (0.5 $\mathrm{mg} / \mathrm{kg}$ bolus followed by $0.2-0.5 \mathrm{mg} / \mathrm{kg} / \mathrm{hr}$ infusion) and dexmedetomidine $(0.1-0.4 \mu \mathrm{g} / \mathrm{kg} / \mathrm{hr})$ had comparable effects on intraoperative TcMEP parameters during stable propofol-opioid anesthesia and suggested that ketamine is the preferred drug for major spinal surgeries. In an experimental study, three groups of rodents received either $75 \mathrm{mg} / \mathrm{kg}$ of intramuscular ketamine followed by IV infusion at $125 \mu \mathrm{g} / \mathrm{kg} / \mathrm{min}$ or $10 \mathrm{mg} / \mathrm{kg}$ IV of propofol followed by IV infusion at $400 \mu \mathrm{g} / \mathrm{kg} / \mathrm{min}$ (standard dose) or $800 \mu \mathrm{g} / \mathrm{kg} / \mathrm{min}$ (high dose). Propofol infusion suppressed the TcMEP amplitude and the amplitude with ketamine was higher than the amplitude when high dose propofol was used. Furthermore, with ketamine, MEP amplitude progressively increased with increasing deep brain stimulation pulse frequency, but no such effect was observed with propofol infusion. These results demonstrated that ketamine increased the amplitude of MEPs with and without a conditioning stimulus [15]. In another study, patients received low-dose ketamine $(0.5 \mathrm{mg} / \mathrm{kg}$ bolus followed by $4 \mu \mathrm{g} / \mathrm{kg} / \mathrm{min}$ infusion) or an equal volume of saline along with stable propofol-remifentanil anesthesia during spine surgery. Ketamine did not increase the minimum voltage needed to elicit maximum amplitude of the MEP response, suggesting that ketamine can be utilized to reduce the dose of propofol and prevent remifentanilinduced acute opioid tolerance via its NMDA receptor 
antagonistic property [8]. In a study by Inoue et al. [16], patients received either $1 \mathrm{mg} / \mathrm{kg} / \mathrm{hr}$ of ketamine or $4 \mathrm{mg} /$ $\mathrm{kg} / \mathrm{hr}$ of propofol as maintenance anesthetic agents along with $50 \% \mathrm{~N}_{2} \mathrm{O}$ in oxygen and $4-5 \mu \mathrm{g} / \mathrm{kg} / \mathrm{hr}$ fentanyl. Amplitudes were significantly higher in the ketamine group compared with that in the propofol group, whereas the latency was similar [16]. Kawaguchi et al. [7] conducted a study to evaluate TcMEPs with ketamine with and without propofol and observed that propofol inhibited TcMEP amplitudes dose-dependently; TcMEP amplitudes recovered only after applying a train of pulses.

Studies using a bolus dose of ketamine are difficult to compare because of variations in doses, administration times, TcMEP evaluation times, stimulation modes, recording duration, and age-groups. Furutani et al. [17] compared the effects of a bolus dose of ketamine $(1 \mathrm{mg} /$ $\mathrm{kg}$ ) versus $0.9 \%$ saline on TcMEP during stable propofolremifentanil anesthesia in female children. Ketamine significantly decreased amplitude and increased relative onset latency compared with saline. However, the suppressive effect of ketamine on TcMEP amplitude lasted only 10 minutes and returned to the approximate baseline value by 30 to 60 minutes after administration. Thus, the authors recommended administering a continuous infusion of ketamine after a bolus dose [17]. In contrast, Ubags et al. [18] reported that a bolus dose of ketamine $(0.5 \mathrm{mg} / \mathrm{kg})$ during opioid-N2O anesthesia did not significantly change TcMEP amplitude and latency in patients (12-57 years) undergoing spinal surgery; the amplitudes increased from a baseline value of $311 \mu \mathrm{V}$ to 349,450 , 387 , and $392 \mu \mathrm{V}$ at $2,5,10,15$ minutes, respectively, after the administration of ketamine. Kalkman et al. [13] demonstrated that a $1 \mathrm{mg} / \mathrm{kg}$ bolus dose of ketamine increased the MEP amplitude within 10 minutes to as high as $150 \%-220 \%$ in three of five human volunteers. Similarly, Yang et al. [19] reported that intraoperative TcMEP monitoring was feasible under ketamine-opioid anesthesia in spinal surgery. Thus, owing to inconsistent results of TcMEP with bolus doses, an infusion dose of any agent should be considered for reliably recording effects on TcMEP parameters.

The 1:4 ketofol dose resulted in a significantly higher MAP at T1-T4 compared with propofol use. Moreover, IV fluids and vasopressor requirements were significantly lower in the ketofol group. These differences may be due to the nearly $50 \%$ lower requirement for propofol in the ketofol group. Propofol causes a dose-dependent suppression of TcMEP amplitudes secondary to inhibition of spinal a motor neurons [20]. Scheufler et al. [21] reported that plasma propofol concentrations between 2 and $6 \mu \mathrm{g} / \mathrm{mL}$ did not significantly affect MEPs. Although plasma concentrations of propofol were not measured in our study, the suppression of TcMEP by propofol was most likely due to sympatholytic effects. In the absence of muscle relaxation, the high dose of propofol required to maintain an immobile patient causes hypotension and interferes with TcMEP signal acquisition. Ketamine maintains hemodynamic stability and tissue perfusion via its sympathomimetic properties; thus, the sympatholytic and sympathomimetic properties of propofol and ketamine, respectively, are balanced in ketofol. This property of ketofol is particularly useful in conditions known to result in weak amplitudes, such as muscle spasticity, preoperative motor dysfunction, children, and concomitant use of inhalational anesthetics [22]. Ketofol is also a very useful drug in hemodynamically unstable patients.

The BIS is a processed electroencephalography variable widely used to guide the administration of hypnotic drugs [23]. BIS values were significantly higher at $\mathrm{T} 1-\mathrm{T} 4$ in group $\mathrm{Y}$ than in group X. However, BIS values remained within the range required for general anesthesia in both groups. Moreover, BIS values at all time points after baseline were similar in group $\mathrm{Y}$. This is attributed to the minimal effects of low-dose ketamine on BIS and is consistent with previous studies $[24,25]$. When ketamine $(1 \mathrm{mg} / \mathrm{kg})$ was administered as a bolus during stable propofol-remifentanil anesthesia, BIS increased from $45 \pm 8$ to $56 \pm 10$, similarly to that in group Y in our study [17].

Ketamine may produce undesirable psychedelic effects, including unpleasant dreaming, extracorporeal experiences, illusions, and hallucinations, during emergence from anesthesia. These effects are often associated with excitement, confusion, euphoria, and fear [26]. However, the psychedelic effects of ketamine are reduced significantly when propofol is administered along with ketamine [7,27]. In our study, 1:4 ketofol was used, and postoperative behavior was normal in all patients; no patients suffered emergence reactions after surgery. These findings are consistent with the study by Guit et al. [27]; TIVA using ketamine $(2 \mathrm{mg} / \mathrm{hr})$ and propofol infusion caused no postoperative psychomimetic effects in patients. Miner et al. [28] also found that the rate of emergence reactions was significantly lower with 1:4 ketofol than with 1:1 ketofol. 
Measurement of TcMEPs is the standard of care during spinal surgeries and is crucial in preventing/limiting new motor deficits. TcMEPs are highly sensitive and specific for predicting postoperative motor deficits [4,29]. However, TcMEP amplitudes are significantly affected by several modifiable factors, such as patient core temperature, systemic blood pressure, oxygenation, ventilation, and the depth and type of general anesthesia [30]. Amplitude changes due to these factors may lead to false positives or negatives, which are undesirable for ideal patient outcomes. These modifiable factors were kept constant in our study, and no significant surgery-related TcMEP alarms developed. Amplitude and BIS values did not change significantly from T1 to T4 during ketofol infusion, indicating stable anesthetic depth critical for accurate neurophysiological interpretation. Moreover, postoperative motor power assessment also demonstrated the absence of any new motor deficits in all cases, suggesting that the intraoperative neurophysiological interpretation under ketofol infusion showed a clinical correlation with the postoperative neurological status.

For patients with preoperative neurological deficits, direct wave (D-wave) monitoring is a viable technique for assessing corticospinal tract function; unlike that in TcMEP monitoring, the activity of the motor tract is directly recorded in D-wave monitoring and is not dependent upon the temporal summation over the anterior motor horn. Similarly to that for TcMEP, the stimulation for eliciting $\mathrm{D}$-wave is given through electrodes placed in the scalp over the putative motor cortex. However, recording is done directly from the spinal cord through the electrodes placed in the subdural or epidural space. Nevertheless, the $\mathrm{D}$-wave amplitude is directly proportional to the number of conducting axons at the recorded level. As such, one cannot record a caudal D-wave if the lesion is below the D9 level. Moreover, D-wave reliability is poor in patients with a history of irradiation and previous surgery. Thus, TcMEP monitoring under ketofol anesthesia is a better technique for lower thoracic and lumbar spine surgeries.

Several limitations of this study should be recognized. The study population was small; future studies should be conducted with larger sample sizes. Ketofol neither increased BIS values beyond 60 nor resulted in psychedelic effects in any patient. However, higher concentrations of ketamine in ketofol may not produce the same results. The plasma concentrations of the study drugs were not deter- mined owing to feasibility issues; future studies should consider measuring plasma drug concentrations when measuring the effects on TcMEPs.

\section{Conclusions}

In our study, TcMEPs were recorded successfully in all patients. Under comparable BIS values and no muscle relaxant use during the maintenance of general anesthesia, we found that ketofol facilitates TcMEPs amplitudes without affecting latency. Ketofol use resulted in better and more stable hemodynamic profiles than did propofol use. Moreover, adverse sequelae like psychedelic effects were not observed. Thus, ketofol can be used effectively and safely in spine surgery with TcMEP monitoring.

\section{Conflict of Interest}

No potential conflict of interest relevant to this article was reported.

\section{ORCID}

Ankur Khandelwal: https://orcid.org/0000-0002-7915-9158; Arvind Chaturvedi: https://orcid.org/0000-0001-7825-8093; Navdeep Sokhal: https://orcid.org/0000-0003-1744-2190; Akanksha Singh: https://orcid.org/0000-0002-8580-2330; Hanjabam Barun Sharma: https://orcid.org/0000-00026910-7573

\section{Author Contributions}

Conceptualization, data curation, writing-original draft preparation: AK; methodology: AK, AC, NS, AS, HBS; project administration, supervision: AC, NS, AS; writingreviewing and editing: AC, NS, AS, HBS; formal analysis: HBS; and all authors approved the final article.

\section{References}

1. Gonzalez AA, Jeyanandarajan D, Hansen C, Zada G, Hsieh PC. Intraoperative neurophysiological monitoring during spine surgery: a review. Neurosurg Focus 2009;27:E6.

2. Rabai F, Sessions R, Seubert CN. Neurophysiological monitoring and spinal cord integrity. Best Pract Res Clin Anaesthesiol 2016;30:53-68. 
3. Calancie B, Molano MR. Alarm criteria for motorevoked potentials: what's wrong with the "presenceor-absence" approach? Spine (Phila Pa 1976) 2008;33:406-14.

4. Kelleher MO, Tan G, Sarjeant R, Fehlings MG. Predictive value of intraoperative neurophysiological monitoring during cervical spine surgery: a prospective analysis of 1055 consecutive patients. J Neurosurg Spine 2008;8:215-21.

5. Pechstein U, Nadstawek J, Zentner J, Schramm J. Isoflurane plus nitrous oxide versus propofol for recording of motor evoked potentials after high frequency repetitive electrical stimulation. Electroencephalogr Clin Neurophysiol 1998;108:175-81.

6. Scheufler KM, Zentner J. Total intravenous anesthesia for intraoperative monitoring of the motor pathways: an integral view combining clinical and experimental data. J Neurosurg 2002;96:571-9.

7. Kawaguchi M, Sakamoto T, Inoue $S$, et al. Low dose propofol as a supplement to ketamine-based anesthesia during intraoperative monitoring of motorevoked potentials. Spine (Phila Pa 1976) 2000;25:9749.

8. Zaarour C, Engelhardt T, Strantzas S, Pehora C, Lewis S, Crawford MW. Effect of low-dose ketamine on voltage requirement for transcranial electrical motor evoked potentials in children. Spine (Phila Pa 1976) 2007;32:E627-30.

9. Tobias JD, Goble TJ, Bates G, Anderson JT, Hoernschemeyer DG. Effects of dexmedetomidine on intraoperative motor and somatosensory evoked potential monitoring during spinal surgery in adolescents. Paediatr Anaesth 2008;18:1082-8.

10. Sloan TB, Mongan P, Lyda C, Koht A. Lidocaine infusion adjunct to total intravenous anesthesia reduces the total dose of propofol during intraoperative neurophysiological monitoring. J Clin Monit Comput 2014;28:139-47.

11. Lemoel F, Contenti J, Giolito D, et al. Adverse events with ketamine versus ketofol for procedural sedation on adults: a double-blind, randomized controlled trial. Acad Emerg Med 2017;24:1441-9.

12. Ozgul U, Begec Z, Karahan K, et al. Comparison of propofol and ketamine-propofol mixture (ketofol) on laryngeal tube-suction II conditions and hemodynamics: a randomized, prospective, double-blind trial. Curr Ther Res Clin Exp 2013;75:39-43.
13. Kalkman CJ, Drummond JC, Patel PM, Sano T, Chesnut RM. Effects of droperidol, pentobarbital, and ketamine on myogenic transcranial magnetic motor-evoked responses in humans. Neurosurgery 1994;35:1066-71.

14. Lam S, Nagata M, Sandhu SK, Veselis RA, McCormick PJ. Effect of ketamine on transcranial motorevoked potentials during spinal surgery: a pilot study. Br J Anaesth 2019;123:e530-2.

15. Furmaga H, Park HJ, Cooperrider J, et al. Effects of ketamine and propofol on motor evoked potentials elicited by intracranial microstimulation during deep brain stimulation. Front Syst Neurosci 2014;8:89.

16. Inoue S, Kawaguchi M, Kakimoto M, et al. Amplitudes and intrapatient variability of myogenic motor evoked potentials to transcranial electrical stimulation during ketamine/N2O- and propofol/ N2O-based anesthesia. J Neurosurg Anesthesiol 2002;14:213-7.

17. Furutani K, Deguchi H, Matsuhashi M, Mitsuma Y, Kamiya Y, Baba H. A bolus dose of ketamine reduces the amplitude of the transcranial electrical motor-evoked potential: a randomized, double-blinded, placebo-controlled study. J Neurosurg Anesthesiol 2019 Oct 16 [Epub]. https://doi.org/10.1097/ANA.0000000000000653.

18. Ubags LH, Kalkman CJ, Been HD, Porsius M, Drummond JC. The use of ketamine or etomidate to supplement sufentanil/N2O anesthesia does not disrupt monitoring of myogenic transcranial motor evoked responses. J Neurosurg Anesthesiol 1997;9:228-33.

19. Yang LH, Lin SM, Lee WY, Liu CC. Intraoperative transcranial electrical motor evoked potential monitoring during spinal surgery under intravenous ketamine or etomidate anaesthesia. Acta Neurochir (Wien) 1994;127:191-8.

20. Liu HY, Zeng HY, Cheng H, Wang MR, Qiao H, Han RQ. Comparison of the effects of etomidate and propofol combined with remifentanil and guided by comparable BIS on transcranial electrical motorevoked potentials during spinal surgery. J Neurosurg Anesthesiol 2012;24:133-8.

21. Scheufler KM, Reinacher PC, Blumrich W, Zentner J, Priebe HJ. The modifying effects of stimulation pattern and propofol plasma concentration on motorevoked potentials. Anesth Analg 2005;100:440-7.

22. Stoicea N, Versteeg G, Florescu D, et al. Ketaminebased anesthetic protocols and evoked potential 
monitoring: a risk/benefit overview. Front Neurosci 2016;10:37.

23. Johansen JW. Update on bispectral index monitoring. Best Pract Res Clin Anaesthesiol 2006;20:81-99.

24. Faraoni D, Salengros JC, Engelman E, Ickx B, Barvais L. Ketamine has no effect on bispectral index during stable propofol-remifentanil anaesthesia. $\mathrm{Br} \mathrm{J}$ Anaesth 2009;102:336-9.

25. Nonaka A, Makino K, Suzuki S, et al. Low doses of ketamine have no effect on bispectral index during stable propofol-remifentanil anesthesia. Masui 2012;61:364-7.

26. White PF, Way WL, Trevor AJ. Ketamine: its pharmacology and therapeutic uses. Anesthesiology 1982;56:119-36.

27. Guit JB, Koning HM, Coster ML, Niemeijer RP, Mackie DP. Ketamine as analgesic for total intravenous anaesthesia with propofol. Anaesthesia 1991;46:24-7.
28. Miner JR, Moore JC, Austad EJ, Plummer D, Hubbard L, Gray RO. Randomized, double-blinded, clinical trial of propofol, 1:1 propofol/ketamine, and 4:1 propofol/ketamine for deep procedural sedation in the emergency department. Ann Emerg Med 2015;65:479-88.

29. Quinones-Hinojosa A, Lyon R, Zada G, et al. Changes in transcranial motor evoked potentials during intramedullary spinal cord tumor resection correlate with postoperative motor function. Neurosurgery 2005;56:982-93.

30. Gruenbaum BF, Gruenbaum SE. Neurophysiological monitoring during neurosurgery: anesthetic considerations based on outcome evidence. Curr Opin Anaesthesiol 2019;32:580-4. 